DOI: $10.21892 / 978-958-5547-34-6.1$

\title{
DESARROLLO PSICOAFECTIVO Y SALUD MENTAL INFANTIL ${ }^{1}$
}

\author{
Viana Ángela Bustos Arcón ${ }^{2}$ \\ Ana Rita Russo de sSánchez ${ }^{3}$ \\ Universidad del Norte
}

\section{Resumen}

El periodo infantil es uno de los más importantes del desarrollo humano, en tanto que, es allí donde se cimentan las bases de la personalidad y la salud mental de los individuos. La Organización Mundial de la Salud (2018) ha señalado la crítica situación de la salud mental en el mundo, adicionalmente, establece que los trastornos mentales se manifiestan a final de la adolescencia (antes de los 14 años) y a principios de la edad adulta, lo cual advierte acerca del impacto de las experiencias tempranas y la psicopatología en el adulto. Considerando la importancia de este planteamiento se realizó la investigación con el objetivo de comprender la relación existente entre el desarrollo psicoafectivo y la salud mental infantil. El estudio tiene como método el análisis de contenido (Valbuena, 2013; Piñuel, 2002) desde una perspectiva psicodinámica. Los resultados indican que se requiere que la salud mental sea comprendida como efecto del desarrollo infantil (Bustos y Russo, 2018) y su atención ha de centrarse en una perspectiva psicoafectiva del desarrollo.

Palabras clave: trastornos mentales, desarrollo infantil, salud mental, infancia, personalidad.

1 Texto derivado de la tesis doctoral: "Evaluación de un programa de intervención psicológica para la recuperación psicoafectiva aplicado a niños y niñas de 6 a 12 años con interferencias en el desarrollo", Universidad del Norte.

2 Becaria Colciencias-Uninorte-Doctorado en Psicología, Universidad del Norte. Magíster en Psicología, Universidad del Norte. Especialista en Psicología Clínica, Universidad del Norte. Representante del Campo de Psicología Clinica, Zona Norte, Colpsic. Miembro Internacional de APA y Miembro Profesional de SIP. Correo: bustosv@uninorte.edu.co.

3 Doctora en Filosofía y Ciencias de la Educación, Universidad de Salamanca (España). Directora de la Maestría en Psicología, Universidad del Norte. Creadora y directora del Programa de Desarrollo Psicoafectivo y Educación Emocional, Universidad del Norte. Correo: arusso@uninorte.edu.co. 


\section{Abstract}

Childhood is one of the most important periods in human development, as the foundations of the personality and mental health of individuals are laid in the bases founded during those years. The World Health Organization (2018) has pointed out the critical situation of mental health in the world, additionally, it establishes that mental disorders manifest themselves at the end of adolescence (before age 14) and at the beginning of adulthood, which warns about the impact of early experiences and psychopathology in adults. Considering the importance of this approach, research was carried out with the objective of understanding the relationship between psycho-affective development and children's mental health. The study has the method of content analysis (Valbuena, 2013; Piñuel, 2002) from a psychodynamic perspective. The results indicate that mental health is required to be understood as an effect of child development (Bustos and Russo, 2018) and its attention must focus on a psychoaffective perspective of development.

Keywords: child development, mental health, childhood, personality. 


\section{Introducción}

La época actual se caracteriza por una intensa hipermodernidad; la tecnología, el uso de las redes sociales y el conocimiento están al alcance de todos, las personas se ven cada día más absortas en los contratiempos diarios y en la preocupación de cubrir las necesidades económicas. La falta de empleo, estudios y oportunidades evidencia problemas sociales, políticos y económicos presentes en el mundo. Estas situaciones han afectado las dimensiones del desarrollo humano, y en particular de la infancia, pues han transformado las condiciones que permiten su despliegue.

La infancia es considerada como el momento evolutivo más importante del desarrollo humano, pues es allí donde tienen lugar los procesos más significativos del individuo, de acuerdo con el crecimiento y la personalidad. Según el Fondo de las Naciones Unidas para la Infancia (Unicef, 2004) la población infantil representa un objetivo primordial en sus investigaciones, proyectos y programas, debido a la naturaleza de sus potencialidades. Es decir, que lo realmente indispensable es el favorecimiento de las más altas garantías para que las potencialidades del desarrollo durante la infancia puedan tener lugar, desde la alimentación hasta lo psicoafectivo.

De este modo, según la citada organización desde que se aprobó la Convención de los derechos del niño se han producido avances notables en el cumplimiento de los derechos de los niños y las niñas, en relación la salud y la educación, mediante la prestación de bienes y servicios esenciales, y un reconocimiento cada vez mayor de la necesidad de crear un entorno protector que les defienda contra la explotación, el abuso y la violencia.

No obstante, los niños, niñas y adolescentes siguen siendo víctimas de problemáticas diversas que desfavorecen el curso esperado del desarrollo, limitando el despliegue de sus potencialidades y la de sus familias. Lamentablemente, en muchos casos, son sometidos a situaciones adversas que ponen a prueba sus recursos afectivos, psíquicos, cognitivos y sociales -por el carácter negativo de esos eventos-, por ello, urge atender los efectos de las situaciones adversas durante la infancia, pues, en ella, el niño/a crece y desarrolla sus habilidades emocionales, cognitivas y sociales, se relaciona con el mundo, con la cultura, así lo señalan Romo y Patiño (2014, p. 69): Es vital comprender "la importancia de las experiencias tempranas y su impacto en la psicopatología del adulto", razón por la que no han sido 
pocos los esfuerzos internacionales, y en particular de Colombia, en favor de leyes, decretos y estándares de educación, entre otros para lograr tal cometido.

En este orden de ideas, en el Plan de acción sobre salud mental 20132020 de la Organización Mundial de la Salud (OMS, 2013) se precisa favorecer acciones específicas para la atención en materia, "estrategias eficaces de intervención para promover la salud mental, prevenir los trastornos mentales, tratar y atender a los afectados y hacer posible su recuperación" (p. 19). Es crucial para la disciplina psicológica y desde los distintos enfoques de la psicología clínica se interroguen además de los hechos clínicos, la importancia de desarrollar acciones que permitan atender el desarrollo psicoafectivo cuando este ha sido afectado como resultado de interferencias en el desarrollo como: abuso, abandono, negligencia, desplazamiento.

La OMS (2001) en su concepto de salud reconoce la condición biopsicosocial de los seres humanos, y la relación que dichos factores tienen en beneficio o no de la salud en sí misma. Es decir, que la salud en general es un conjunto de condiciones positivas, individuales y contextuales de los individuos que favorecen el desarrollo de sus potencialidades. Así mismo, la salud mental es entendida como "un estado de bienestar en el que la persona materializa sus capacidades y es capaz de hacer frente al estrés normal de la vida, de trabajar de forma productiva y de contribuir al desarrollo de su comunidad" (OMS, 2013, p. 42), hace referencia al despliegue de las potencialidades humanas, a la vitalidad individual y a las relaciones sociales. Es importante, reconocer en esta definición, que la salud mental implica también afrontar la adversidad, o sea, las dificultades, ansiedades o conflictos que hacen parte de la vida a lo largo del ciclo vital, y que no constituyen en sí mismo problemas o trastornos emocionales.

En este sentido, se entiende que la salud mental-como "una parte indivisible de la salud general contribuye a las funciones de la sociedad y tienen un efecto sobre la productividad general" (OMS, 2004, p. 23), porque atañe al desarrollo en sí mismo, y a la materialización de las potencialidades y capacidades humanas en una relación directa con la comunidad, la sociedad y la cultura. Por lo tanto, es indispensable para el desarrollo del país en todos sus ámbitos.- 
Dada la naturaleza de la salud mental, su atención y seguimiento se complejizan. Son innegables los logros de la ciencia médica y las ciencias humanas, en especial la disciplina psicológica en lo que respecta a la atención en salud y enfermedad (vacunas, medicamentos, tratamientos, psicoterapia, programas de atención en salud, protocolos de atención, pruebas, test psicológicos, etc.), que han permitido a las sociedades disponer de mejores instrumentos y recursos para la atención en materia de salud y enfermedad.

Las cifras generales en materia de salud mental se consideran preocupantes, por ejemplo, la OMS (2018) estima que cerca de 300 millones de personas en el mundo padecen de depresión o trastornos bipolares. Esto evidencia la necesidad de mantener los esfuerzos mancomunados de todos los sectores posibles para seguir acompañando la promoción, prevención e intervención en salud mental.; de este modo, es necesario-atender estas situaciones desde las ciencias de la salud y las ciencias humanas, la investigación científica, la cooperación internacional, participación sectorial, la sociedad civil y la política desarrollando acciones y técnicas destinadas a la promoción y prevención en salud, pero muy especialmente a la detección temprana de las afectaciones mentales en los individuos.

Por lo tanto, si se comprende que "las actividades de promoción de salud mental implican la creación de condiciones individuales, sociales y ambientales que permitan el óptimo desarrollo psicológico y psicofisiológico" (OMS, 2004, p. 17), cuyo objetivo es "ejercer un impacto sobre los determinantes de la salud mental con el fin de aumentar la salud mental positiva" (OMS, 2004, p. 17). No obstante, solo será posible favorecer la salud mental al advertir las dimensiones que comprenden a los seres humanos como una totalidad, o sea, sus condiciones biopsicosociales y emocionales particulares, pero a la vez integradas. En este sentido, se considera que la promoción en salud y la prevención en salud mental, se encuentran íntimamente relacionada, puesto que la promoción del desarrollo biopsicosocial, de individuos y/o comunidades, incide directamente en la prevención de trastornos mentales.

Sería correcto afirmar: "Cuando la promoción de la salud mental tiene el objeto de mejorar la salud mental positiva en la comunidad, también podrá tener el resultado secundario de disminuir la incidencia de los trastornos mentales" (OMS, 2004, p. 18). Así, "la salud mental positiva actúa como un poderoso factor de protección contra las enfermedades 
mentales" (OMS, 2004, p. 18); y en general, cuanto más se favorece el desarrollo humano y las potencialidades humanas más se promociona la salud mental y se previenen trastornos mentales en las personas, al igual que en las comunidades.

Es fundamental comprender que la salud y la salud mental es el resultado de las relaciones posibles entre todos los factores que participan en el desarrollo humano, lo biológico, lo psicológico, lo psíquico y lo social. El desarrollo humano es "un proceso de co-construcción entre los factores constitucionales y adquiridos en el devenir evolutivo del individuo. Dichos factores van sucediendo en un continuo relacional en compañía de padres, sustitutos, pares, maestros, escuela y comunidad" (Bustos y Russo, 2018, p. 2). Por ello, es mucho más urgente comprender que "la perfección tiene que ver con las máquinas, y las imperfecciones que son características de la adaptación humana a la necesidad constituyen una cualidad esencial del ambiente que facilita" (Winnicott, 1968, p. 216), es decir, que la salud no es un esquema, esto implica que no es universal, y por ende es singular.

La salud es en esencia una particularidad, pues teniendo en cuenta las condiciones del desarrollo emocional de los individuos y la subjetividad que transciende a cada ser humano, el resultado de lo que se denomina salud mental dependerá del contexto de cada individuo y sus peculiaridades históricas, que supone pensar que las acciones de los Estados deben estar dirigidas a la intervención psicológica de las personas y comunidades, especialmente en materia de atención, cuidado y recuperación de niños, niñas y adolescentes, desde una perspectiva evolutiva, histórica y clínica.

La intervención temprana en salud se refiere a un modelo de salud que supera las acciones de promoción de la salud y prevención de la enfermedad, enfatizando la necesidad de dar solución a situaciones complejas de la salud en los individuos y su comunidad. La OMS (2013) promueve el diseño y aplicación de estrategias que "mediante la detección precoz, prevención y tratamiento de los problemas afectivos o de conducta, sobre todo en la infancia y la adolescencia" (p. 18) respondan a la solución de problemáticas relacionadas con la salud mental; pues es de recordar, el lugar privilegiado que ocupa la infancia en el panorama mundial. Lo anterior, alerta sobre la necesidad de los niños, niñas y adolescentes de hacer frente a las complejidades culturales, sociales, económicas y políticas que afrontan, la vulnerabilidad en la que se encuentran (tanto la infancia 
como la adolescencia), por lo que es una urgencia favorecer el desarrollo de las potencialidades y salud mental en todos los escenarios posibles, en condiciones positivas y en la adversidad.

\section{Salud mental y su detección temprana en la infancia}

El Fondo de las Naciones Unidas para la Infancia (2004) establece que la infancia se define en función a la calidad en que transcurre su vida, precisa con ello que la prioridad es la constitución de las potencialidades humanas; tal condición de calidad, se ve comprometida, en razón de la vulnerabilidad que aún se encuentra en la infancia, desde las dificultades en el acceso al agua potable, la educación, los servicios de salud, hasta los obstáculos para el curso esperado del desarrollo psicoafectivo.

Son reconocidos los logros alcanzados por las naciones en el mundo en materia de protección de los derechos en la infancia, empero, el Fondo de las Naciones Unidas para la Infancia (Unicef, 2012) expresa su preocupación por la población infantil, debido a las adversas circunstancias que los niños y niñas enfrenta alrededor del mundo, particularmente los referidos al acceso a servicios de salud, agua potable, educación y al trabajo al que son sometidos, advirtiendo que: "Ofrecer a todos los niños y niñas el mejor comienzo en la vida" (Unicef, 2014, p. 1) debe ser la prioridad. Lo anterior, convoca a considerar la urgencia de acciones centradas en la promoción, prevención e intervención en la infancia para asegurar el adecuado despliegue de las potencialidades humanas tempranamente.

Resulta oportuno destacar que las acciones ya no solo deben dirigirse a la promoción en salud y prevención de la enfermedad, sino a un estatuto superior, es decir, la detección temprana y la intervención específica. La OMS (2013) promueve el "diseño y el establecimiento de sistemas de salud mental y apoyo psicosocial que faciliten la resiliencia de la comunidad y ayuden a la población a afrontar las emergencias humanitarias" (p. 4l), en particular de la infancia, atendiendo las necesidades en beneficio de la recuperación psicoafectiva en situaciones de vulnerabilidad o adversidad, comprendiendo acciones centradas en la detección temprana e intervención específica. 
En el caso específico de la recuperación psicoafectiva en la infancia, la Convención de Derechos del Niño (1989) estableció que es indispensable "promover la recuperación física y psicológica y la reintegración social de todo niño víctima de: cualquier forma de abandono, explotación o abuso; tortura u otra forma de tratos o penas crueles, inhumanos o degradantes; o conflictos armados" (Unicef, 1989, artículo 39, p. 36). Se requieren acciones de detección temprana e intervención específica cuando las potencialidades de los niños y niñas se encuentren comprometidas en situaciones de interferencias en el desarrollo. Apremia contar con programas de intervención para la recuperación psicoafectiva dirigidos a la población infantil que permitan favorecer el curso normal del desarrollo de sus potencialidades, maduración, adaptación, y desarrollo emocional.

Para ello, es indispensable comprender que "las pautas básicas se establecen durante el proceso de maduración emocional, y los factores más influyentes son los que actúan al comienzo" (Winnicott, 1970a, p. 48-49). O sea, que lo primordial es el cuidado de la infancia, atender sus necesidades vitales y conflictos propios de los momentos evolutivos en el curso esperado del desarrollo y en casos de interferencias en el desarrollo. Teniendo en cuenta, además, que la infancia supone un momento cumbre del desarrollo de todo ser humano, acuciante por sus condiciones y experiencias directas de crecimiento y adaptación.

Más abiertamente es preciso comprender que "la salud mental no depende solo de la herencia ni de acontecimientos fortuitos, sino que sus fundamentos se construyen en forma activa en la primera infancia" (Winnicott, 1965, p. 206). Es decir, que cuanto más se cuide de la infancia, y se asistan sus necesidades, mayores posibilidades de salud mental habrá en las generaciones futuras, pues, la infancia requiere de condiciones biopsicosociales adecuadas para el óptimo despliegue de sus potencialidades humanas. Asimismo, la integración de todo niño a un entorno familiar que aporte a su crecimiento psicoafectivo. Es importante destacar que toda familia implica urgencias y conflictos emocionales que deben ser atendidos con base en las atribuciones subjetivas que el niño va construyendo.

El Ministerio de Salud y Protección Social (2015) advierte que "los datos epidemiológicos hasta el 2005 sugerían una prevalencia de trastornos mentales hasta del 20\% de los niños y adolescentes; de estos, entre un 4\% y un $6 \%$ requieren atención en servicios de salud" (p. 206), al tiempo que 
"para la población infantil de 7 a 11 años se incluyen: depresión mayor, ansiedad de separación, trastorno de ansiedad generalizada, trastorno negativista desafiante, trastorno por déficit de atención e hiperactividad (TDAH) y trastorno de la conducta" (Minsalud, 2015, p. 206), lo que implica que en el caso de la infancia, en particular en el caso de interferencias en el desarrollo, la recuperación y reintegración "se llevarán a cabo en un ambiente que fomente la salud, el respeto de sí mismo y la dignidad del niño" (UNICEF, 1989, artículo 39, p. 36).

En relación a la Encuesta Nacional de Salud Mental (Minsalud, 2015), estudios científicos recientes del Programa Desarrollo Psicoafectivo y Educación Emocional de la Universidad del Norte, a saber: Resultados de la implementación del Programa de Desarrollo Psicoafectivo y Educación Emocional en la Primera Infancia (2013-2014), y Estudio longitudinal para el diseño del programa de intervención para la Recuperación Psicoafectiva (2014) ponen en evidencia las interferencias en el desarrollo más frecuentes en Colombia, y que corresponden a: agresión-maltrato (35\%), abandononegligencia (27\%), desplazamiento forzoso por violencia (23\%) y abuso sexual (15\%); lo que obliga a la disciplina psicológica a atender las necesidades reales de cuidado y protección del desarrollo emocional en la infancia y el despliegue de las potencialidades humanas.

\section{Infancia, salud psíquica y desarrollo psicoafectivo}

Es claro hasta aquí, que la infancia ocupa un lugar importante en el desarrollo de las sociedades. Los esfuerzos de los Estados se centran en atender sus necesidades y en proteger sus derechos, pues también es allí donde habita lo primordial en materia de salud mental. Como bien señala Freud (1979) la infancia es el escenario de la "maduración, adaptación y estructuración" (p. 65), por esto su especial cuidado, pues es el resultado de los procesos madurativo-constitucionales (Greenspan, 1992) que determinarán las condiciones de la salud mental. El desarrollo "denota los procesos vinculados temporalmente, los cambios progresivos del funcionamiento adaptativo" (Tamez, Ortega, Roa, Russo, Galindo, 2006, p. 15); es decir, el desarrollo es de carácter progresivo e inagotable. Winnicott (1967) señala una característica de la salud, y es que: "(...) nunca deja de desarrollarse", por tanto, si el desarrollo es progresivo e inagotable, la salud lo es de igual manera. 
Pues bien, es necesario considerar la salud en términos psíquicos. En este sentido, "el crecimiento no es una simple tendencia heredada, sino, además, un entrelazamiento de suma complejidad con el ambiente facilitador" (Winnicott, 1968, p. 223), lo que da lugar durante la infancia a transformaciones psíquicas vitales a partir de los conflictos específicos de acuerdo con los momentos del desarrollo (Phillips, 1998; Rangel, 2009; Guillerault, 2009; Serguéyevna, 2015). De acuerdo con Stolorow y Atwood (así como es citado por Ávila, 2009, p. 4) se refiere a la salud psíquica como "la habilidad que tiene la persona saludable para lograr un balance óptimo entre el mantenimiento de su organización psicológica, por un lado, y su apertura por otro a nuevas formas de experiencia". Este balance se tiene lugar durante la infancia cuando el niño/a desarrolla recursos psicológicos, relacionales y de adaptación.

Siendo que "Freud, Klein, Bowlby, Jung, Erikson, Piaget y Vygotsky, (...) han dejado una indeleble huella en el entendimiento actual de como el humano vive y experimenta la salud mental y cómo la pierde" (Romo y Patiño, 2014, p. 68). El estudio de la salud mental y del desarrollo "(...) debe ir más allá del conteo de frecuencias y de la descripción detallada de síndromes y trastornos en las etapas discretas de la vida." (Romo, Patiño, 2014, p. 68), se debe profundizar en las complejidades de lo psicoafectivo; en la salud emocional y los aspectos biopsicosociales como un todo, como una unidad y la comprensión de la salud mental como una condición particular (Duro, 2012).

De lo expuesto, se puede inferir que el desarrollo psicoafectivo es el resultado de las complejidades de lo biopsicosocial, frente a lo que es vital "conocer las tensiones que son inherentes a la vida y los medios que se emplean para hacerles frente" (Winnicott, 1961, p. 20). El desarrollo psicoafectivo es un modelo integral, conjuga las condiciones biopsicosociales, dando un especial valor a lo psíquico, y a los conflictos vitales del crecimiento. "Las fuerzas de la vida, hacia la integración de la personalidad, hacia la independencia, son extremadamente poderosas y, si las condiciones son buenas, el niño progresa (...) Nuestra concepción del desarrollo es dinámica" (Winnicott, 1970b, p. 20). Se trata de otorgar valor al modo como cada niño crece y despliega sus propias capacidades.

La integración es la característica principal del desarrollo psicoafectivo, y por ende, de la salud mental, y la base de esta, a su vez, "La base de la 
salud mental es una personalidad bien integrada" (Klein, 1960, p. 272), o bien, "a lo que sucede en las etapas tempranas del desarrollo de la personalidad. (...) es integración, aplicable a casi todas las tareas evolutivas" (Winnicott, 1967, p. 35). El desarrollo psicoafectivo es el resultado de los procesos madurativo-constitucionales y relacionales, o sea "los procesos de la evolución mental y su comprensión de la interacción entre las fuerzas externas e internas que forman la personalidad del individuo" (Freud, 1979, p. 11), referidos a la "red de impulsos, afectos, relaciones objetales, aparatos del yo, con sus funciones y defensas" (Freud, 1979, p. 12), esto permite que los conflictos vitales transcurran de forma esperada, dando lugar a nuevos y más complejos procesos mentales y relaciones con el mundo interno y externo, pues como señala Blos (2011) "cada fase del desarrollo contribuye de manera característica al desenvolvimiento de la personalidad" (p. 35) y conduce a formas más integradas de la vida mental, que resultan en la adaptación y la consolidación de la personalidad. Por lo que el desarrollo psicoafectivo supone más una integralidad de elementos dialécticos y no lineales o simplemente cronológicos.

En consecuencia, el desarrollo psicoafectivo pleno da lugar a las condiciones de la salud mental desde la infancia, pues al conjugar las dimensiones biopsicosociales del niño/a los conflictos vitales se resuelven en asocio con el ambiente facilitador, que impulsan la adaptación y los cimientos de la personalidad. Cuando más favorable son las condiciones biopsicosociales, mejor es el tránsito durante la infancia de las necesidades, demandas y conflictos de cada periodo evolutivo. Mientras que, cuando alguna o varias dimensiones del desarrollo psicoafectivo falla, las complejidades de los procesos madurativo-constitucionales se entorpecen, retrasando el desplieguen esperado del desarrollo, y los conflictos vitales, la adaptación y la personalidad se obstaculizan, generando entonces una interferencia en el desarrollo que, de no ser atendida en el momento evolutivo, tendría consecuencias desfavorables en el desarrollo y en la salud mental.

\section{Interferencias en el desarrollo y Recuperación Psicoafectiva}

El concepto de recuperación psicoafectiva, si bien no pertenece al constructo teórico de la psicología dinámica, su función está dirigida a superar los embates del trauma. De acuerdo con el Diccionario de 
psicoanálisis de Laplanche y Pontalis (1996), el trauma se define como "un acontecimiento de la vida del sujeto caracterizado por su intensidad, la incapacidad del sujeto de responder a él adecuadamente y el trastorno y los efectos patógenos duraderos que provoca en la organización psíquica" (p. 447). O como expresa Winnicott (1967): "Un trauma representa la ruptura de la continuidad de la línea de la existencia del individuo" (p. 28). Por tanto, la complejidad del desarrollo psicoafectivo y el desarrollo emocional en particular permitiría aportar a la atención de fenómenos sociales como el abuso, el maltrato, la negligencia, el abandono, etc., que afectan de forma directa los procesos psicoafectivos en la infancia.

La psicología dinámica comprende las condiciones psíquicas y relacionales de los individuos desde su nacimiento en función del desarrollo emocional y la personalidad. Winnicott (1965) señala que su interés radica "esencialmente el ser humano: el ser humano en tanto es, siente, actúa, se relaciona y medita" (p. 201). Y es precisamente esto lo que nos conduce a advertir "la división misma de la personalidad en ello, yo y superyó se nos presenta como una estructura psíquica en la cual cada parte tiene sus derivaciones, sus alianzas, sus fines y su modo de funcionamiento específicos" (Freud, 1979, p. 15), siendo esto lo que otorga una constitución particular e histórica que ha de ser contemplada en la comprensión de la salud mental.

Bajo estas premisas se hizo fundamental conocer el desarrollo infantil, caracterizando los conflictos vitales en los momentos evolutivos, trabajo que autores como los ya citados, Melanie Klein, Anna Freud, Donald Winnicott, estudiaron con rigurosidad, evidenciando las dimensiones del desarrollo emocional de los niños/as y la importancia de este en la personalidad y adultez. De acuerdo con Freud (1979) era necesario:

Rastrear las combinaciones que conducen desde la completa dependencia emocional del niño hasta la comparativa autosuficiencia, madurez sexual y de relaciones objetales del adulto, una línea graduada de desarrollo que provee la base indispensable para la evaluación de la madurez o inmadurez emocional, la normalidad o anormalidad. (p. 55)

Es decir, conocer las características de los procesos anímicos y emocionales de los niños/as permite establecer las condiciones de la adaptación, madurez y la normalidad, o sus interferencias. El objetivo del 
desarrollo psicoafectivo, así como la recuperación psicoafectiva, radica en el "desarrollo emocional del niño y las interferencias con los procesos de maduración provenientes del medio y los conflictos internos" (Winnicott, 1961, p. 270).

Las interferencias en el desarrollo obedecen a situaciones, eventos o condiciones que afectan de forma directa o indirecta el curso esperado del desarrollo, por ejemplo: el abuso, el maltrato, el abandono, la negligencia, el desplazamiento, entre otros. Esto obstaculiza que las líneas del desarrollo, los conflictos vitales, los mecanismos de defensa o las relaciones con el medio puedan transcurrir de acuerdo con las necesidades propias del ciclo vital, y por el contrario, generan dificultades psicoafectivas y emocionales como inhibición, depresión o agresividad, que impiden a corto y a largo plazo una buena salud mental cuando no pueden ser atendidas oportunamente. Es comprensible que "la experiencia indica que los efectos psicológicos son más severos si el trauma es generado por el ser humano, perpetrado por una figura de apego, con crueldad, repetido y en la infancia." (Marrone, citado por Gómez, 2013, p. 133).

El desarrollo psicoafectivo obedece a la progresión, y con ello es posible pensar que aún en los casos de las interferencias es posible direccionar favorablemente todos los procesos del desarrollo; su condición dinámica, así lo evidencia. Winnicott (1963) afirma que existe "la tendencia a la recuperación" (p. 85), es decir, aunque no es posible evitar las adversidades, si es posible transformar sus efectos negativos. Esto permite sostener que en los casos de niños/as en interferencias en el desarrollo, la recuperación psicoafectiva es un proceso emocional posible y una necesidad científica en las ciencias humanas.

No estamos de acuerdo con que la salud sea simplemente la ausencia de trastornos psiconeuróticos (...) podemos afirmar que salud no es comodidad, los temores, los sentimientos conflictivos, las dudas y las frustraciones con tan característicos en la vida de una persona sana como los rasgos positivos. (Winnicott, 1967, p. 34)

Por otra, parte, la OMS (2013) define recuperación en los términos expresados en el siguiente párrafo, aunque deja muy en claro que no se refiere a la curación, sino más bien a la resiliencia: 
La aparición o reaparición de la esperanza, la aceptación de las capacidades y discapacidades propias, el despliegue de una vida activa, la autonomía personal, la identidad social, la asignación de un significado y una finalidad a la vida, y un sentido positivo de sí mismo. (p. 42)

No es posible negar las consecuencias de la adversidad, y en el caso de la infancia mucho menos, aun así, si es posible minimizar las consecuencias negativas de éstas y favorecer el curso esperado del desarrollo psicoafectivo.

Recuperación no es sinónimo de curación. Aquella está vinculada con situaciones internas de las personas que se consideran en recuperación —esperanza, mejoría, autonomía y vinculación- y situaciones externas que la facilitan: el respeto de los derechos humanos, la existencia de una cultura que propicia la mejoría y los servicios de apoyo a la recuperación. (OMS, 2013, p. 42)

La recuperación psicoafectiva al corresponderse con la adversidad en un momento determinado del ciclo vital debe también acogerse a los conflictos, necesidades y potencialidades de los procesos madurativoconstitucionales interrumpidos. Por lo que, "si examinamos en detalle nuestras nociones con respecto a la normalidad descubriremos que esperamos una estrecha correspondencia entre las distintas líneas de desarrollo" (Freud, 1979, p. 71), las interferencias deberán ser atendidas bajo las condiciones mencionadas anteriormente en el desarrollo psicoafectivo (...), de igual modo, los procesos defensivos, que "son aquellos medios psicológicos que el yo utiliza para solucionar los conflictos que surgen entre las exigencias instintivas y la necesidad de adaptarse al mundo de la realidad, bajo determinadas influencias del ambiente familiar y social" (Cárcamo, 1993, p. 9).

Ningún niño está exento de conflictos y obstáculos que debe afrontar a lo largo de su desarrollo, pero es posible, que con su maduración y desarrollo, frente a ellos logre afianzar mecanismos psíquicos, que le permitan asumir y tramitar su historia.

En casos de interferencias en el desarrollo, en particular en la recuperación psicoafectiva, se centrarán en la integración de los ámbitos que 
componen a los seres humanos, es decir lo psicoafectivo, la resignificación y la resiliencia, como bien refiere Winnicott (1970a):

Un tema muy amplio es el de la provisión de oportunidades a los niños para que vivan, tanto en el hogar como en la escuela, y es un axioma que los niños que arriban con facilidad al sentimiento de que existen son los más fáciles de manejar. Son los menos vulnerables frente al embate del principio de realidad. (pp. 63-64)

La recuperación psicoafectiva es indispensable para el favorecimiento del desarrollo en la infancia, bajo un modelo de favorecimiento de la elaboración psíquica, las relaciones con el medio y la personalidad. Es claro que "el conflicto psíquico que impide al sujeto integrar en su personalidad consciente la experiencia que le ha sobrevenido" (Laplanche y Pontalis, 1996, p. 448). Por lo que un programa de recuperación psicoafectiva permitiría hacer frente a los efectos negativos de las interferencias en el desarrollo.

La atención de las interferencias en el desarrollo se centra en el yo, pues es el yo quien afronta la adversidad y dado que se encuentra "en su proceso de formación; no está del todo desarrollado y es, por tanto, débil" (Freud, 1993, p. 156). El yo, sufre las consecuencias de las interferencias en el desarrollo debido a su inmadurez, lógico, pero es en particular, porque el impacto de las interferencias supera las capacidades madurativoconstitucionales del yo durante la infancia, se requiere entonces, de acciones dirigidas a favorecer mecanismos más elaborados de acuerdo con el momento evolutivo y el yo, o quedaría en riesgo la integración necesaria para la buena salud mental.

\section{Metodología}

Siendo que el objetivo es comprender la relación existente entre el desarrollo psicoafectivo y la salud mental durante la infancia a la luz de la teoría psicodinámica, se establecen el diseño y procedimiento, entre otras aspectos de la investigación, como a continuación se desarrollan.

Diseño: El diseño corresponde a la investigación documental, este es un estudio metódico, sistemático y organizado (Uribe, 2013) de 
documentos, textos, artículos y demás escritos sobre un tema (Packer, 2013; Strauss \& Corbin, 2012; Bernal, 2010), dicho método es "una abstracción científica (...) una labor interpretativa (...) según su naturaleza analítica" (Uribe, 2013, p. 198), quiere decir esto: "Un proceso no matemático de interpretación, realizado con el propósito de descubrir conceptos y relaciones en los datos (...) luego organizarlos en un esquema explicativo teórico" (Strauss \& Corbin, 2012, p. 12). En efecto, la revisión documental sostenida aquí, comprende explicaciones teóricas acerca de la relación entre el desarrollo psicoafectivo y la salud mental durante la infancia desde una perspectiva psicodinámica.

Procedimiento: El procedimiento y análisis de los datos obedece a la investigación cualitativa, a la luz de la investigación documental atendiendo a "escritos (artículos, informes, planificadores, etc.), entrevista, observaciones, entre otros" (Valbuena, 2013, p. 214). Los documentos son jerarquizados de acuerdo con las categorías de: desarrollo psicoafectivo y salud mental. El procedimiento para la selección de textos comprende: (a) Definición del objetivo: comprender la relación existente entre el desarrollo psicoafectivo y la salud mental durante la infancia a la luz de la teoría psicodinámica; (b) Selección y jerarquización de textos, documentos, libros, artículos, publicaciones científicas, boletines, etc., en virtud de las categorías de desarrollo psicoafectivo y salud mental; y (c) Relaciones teóricocomprensivas entre desarrollo psicoafectivo temprano y problemáticas de salud mental.

Instrumentos y participantes: Los instrumentos aquí expresados obedecen a las fuentes de consulta, seleccionadas a partir de la teoría psicodinámica, por lo que comprenden una delimitación conceptual referida a: desarrollo psicoafectivo y salud mental. Los textos son de carácter bibliográfico, corresponde a un "análisis de la información escrita sobre un determinado tema, con el propósito de establecer relaciones, diferencias, etapas, posturas (...) del conocimiento respecto al tema objeto de estudio" (Bernal, 2010, p. 111). Los textos escogidos son de carácter comprensivo e interpretativo, sobre el desarrollo psicoafectivo y la salud mental en la teoría psicodinámica.

Siendo este estudio, una investigación documental, no incluye participación de sujetos, el desarrollo del estudio y sus resultados son de carácter conceptual. Podría ser que en el futuro, los alcances conceptuales 
impliquen la participación de sujetos, bajo otras modalidades científicas, metodologías y/o diseños diversos.

Análisis de la información: El análisis de los datos implica "procesar datos relevantes sobre las condiciones mismas en que se han producido aquellos textos, o sobre las condiciones que puedan darse para su empleo posterior" (Piñuel, 2002, p. 2), se obtienen los resultados en forma de exégesis a la luz de la teoría psicodinámica.

\section{Discusión}

La salud mental es un problema actual que implica nuevos retos científicos y aplicados que permitan reducir las consecuencias de los avatares de la vida en una salud mental positiva.

Se estima que la carga debida a los trastornos mentales se incrementará en las próximas décadas. En el 2020 es posible que los trastornos mentales conformen el 15\% de los años de vida perdidos y ajustados por discapacidad. Se espera que la depresión se convierta en la segunda causa más importante de discapacidad en el mundo (Murray \& López, 1996). Los países en vías de desarrollo con un sistema de salud mental poco desarrollado probablemente verán incrementada de forma sustancial la carga atribuida a los trastornos mentales. (...) Se espera que la esperanza de vida de personas con trastornos mentales pueda incrementarse, y que se mejore también la esperanza de vida de un número creciente de personas mayores que sufren depresión y demencia. (OMS, 2003, p. 12)

A esta dura tarea, De la Fuente (2014) explica los retos de la salud mental a nivel global, cuya perspectiva permite centrarse en fortalecer medios y recurso humano y disminuir factores de riesgo: 
Tabla 1

Retos de la salud mental

\begin{tabular}{|c|c|}
\hline 1 & $\begin{array}{l}\text { Identificar las causas, factores de riesgo y factores protectores. } \\
\text { Identificar factores biológicos y sociales modificables } \\
\text { ¿Cuál es el impacto de la pobreza, la violencia, la guerra, la migración? }\end{array}$ \\
\hline 2 & $\begin{array}{l}\text { Avanzar en la prevención e instrumentar intervenciones tempranas. } \\
\text { Reducir el tiempo de enfermos no detectados, no tratados. } \\
\text { Desarrollar intervenciones de prevención primaria } \\
\text { Proteger a la niñez, eliminar el abuso a menores. }\end{array}$ \\
\hline 3 & $\begin{array}{l}\text { Ampliar el acceso a los tratamientos disponibles y fortalecerlos } \\
\text { Detección oportuna en la atención primaria } \\
\text { Reducir el costo de medicamentos } \\
\text { Servicios de rehabilitación en el seno comunitario }\end{array}$ \\
\hline 4 & $\begin{array}{l}\text { Tener claridad sobre el peso de las enfermedades mentales } \\
\text { Eliminar el estigma, la discriminación y la exclusión de los enfermos. } \\
\text { Desarrollar instrumentos y modelos transculturalmente válidos. }\end{array}$ \\
\hline 5 & $\begin{array}{l}\text { Contar con recursos humanos capacitados } \\
\text { Crear centros que integren la enseñanza con la investigación y la atención } \\
\text { Incorporar a la salud mental en el entrenamiento del personal de salud }\end{array}$ \\
\hline 6 & $\begin{array}{l}\text { Transformar los sistemas y las políticas de salud } \\
\text { Incluir a la salud mental en los programas para el desarrollo } \\
\text { Integrar las enfermedades mentales que correspondan al rubro de las } \\
\text { enfermedades crónicas para compartir inversión en investigación e } \\
\text { infraestructura. }\end{array}$ \\
\hline
\end{tabular}

Fuente: Grand Challenges in Global Mental Health. Nature, citado por De La Fuente (2014, p. 8).

Lo anterior comprende las directrices internacionales sobre las cuales deben dirigirse los esfuerzos de atención en materia de salud mental y desarrollo. En este sentido, la prioridad se centra en la infancia y en todo aquello que pueda afectar su curso y el desarrollo de sus potencialidades. Si se piensa en la recuperación psicoafectiva propiamente dicha, puede ubicarse en el objetivo 2 y 3, centrados en Avanzar en la prevención e instrumentar intervenciones tempranas y Ampliar el acceso a los tratamientos disponibles y fortalecerlos (De La Fuente, 2014).

El bienestar físico, psicológico y social, que va más allá del esquema biomédico, donde abarca la esfera subjetiva y del comportamiento del ser humano. No es la ausencia de alteraciones y de enfermedad, sino un concepto positivo 
que implica distintos grados de vitalidad y funcionamiento adaptativo. (Oblitas, 2004, p. 3)

Los esfuerzos deben centrarse en la actualidad a recuperar la vitalidad humana en general, y en la infancia a propiciar la resiliencia frente a la adversidad en casos de interferencias en el desarrollo, comprendiendo que "La resiliencia, es la forma como estos individuos reaccionan a esta pérdida y se ajustan a la situación, lo cual determina el impacto final sobre el bienestar" (DPN, 2007, p. 7).

Las investigaciones en psicología evolutiva y los grandes teóricos del desarrollo concuerdan en que las experiencias tempranas son fundamentales en la constitución de la mente, pues esta se construye en relación con un otro, quien puede fortalecerla a través de un lazo satisfactorio o, por el contrario, destruirla. (Freud; Fonagy; Vigotsky; Bowlby; Stern; Winnicott; citados por Fossa, 2013, p. 151)

Si se considera estructuralmente frágil a la infancia (Cohen, 2015), entonces, es allí a donde se deben dirigir los esfuerzos para el adecuado desarrollo de su personalidad, la vivencia sin tensiones de las ansiedades esperadas de acuerdo con las condiciones evolutivas, al fomentar un yo libre de conflicto, capaz de sobreponerse a la adversidad y lidiar con los avatares normales de la vida. Winnicott (1951) a propósito de la salud mental advierte luego de la segunda sesión del Comité de Expertos en Salud Mental de la OMS advierte la importancia de que:

Se ocupe principalmente del manejo de la infancia y la niñez, dando por sentado algo que los médicos quizá no habrían aceptado cincuenta años atrás: las bases de la salud mental del adulto se echan en su infancia y niñez y, por supuesto, en su adolescencia. (p. 197)

Mientras Bowlby (1951) en esa misma época, y siendo asesor de salud mental de la OMS, señala que la relación madre-hijo es de extremo cuidado y que requiere de una atención especial. Y advierte muy tempranamente, que las alteraciones de esta relación pueden producir graves complicaciones emocionales. Su descripción, aunque destinada al vínculo madre-hijo, es perfecta para atender de forma precisa las interferencias en el desarrollo. 
Esas criaturas no son pizarrones de los que se puede borrar el pasado con un plumero o una esponja, sino seres humanos que llevan consigo sus experiencias previas y cuya conducta actual se ve profundamente afectada por los sucesos pretéritos. (Bowlby, citado por Winnicott, 1951, p. 198)

Desde la psicología dinámica, se entiende la salud como especialmente emocional, por tanto, cuando más favorable es el curso esperado del desarrollo, en sus dimensiones integradas, se gozará en los momentos evolutivos de mayor salud. Por lo que, a esta ardua tarea, la psicología dinámica propone un modelo de recuperación psicoafectiva basado en el fortalecimiento del yo de niños/as en interferencias en el desarrollo, con el propósito de atender las consecuencias psicoafectivas en casos de maltrato infantil.

La discusión aquí propuesta no está terminada. Ocupa, por el contrario, la necesidad de revisar el abordaje que se hace en la actualidad en materia de salud mental y el desarrollo psicoafectivo. Sin embargo, las contribuciones teóricas que se proponen permiten repensar los fenómenos actuales a través de la mirada de la integralidad afectiva vital en la consecución de la salud mental temprana.

Es obligación disciplinar disponer de los recursos científicos, éticos e investigativos en el favorecimiento del desarrollo humano, especialmente en casos de poblaciones vulnerables. La coyuntura nacional convoca a proponer nuevas y renovadas estrategias de atención a los problemas de salud mental en la infancia, y de promover en la disciplina psicológica un compromiso audaz en el uso de nuevas tecnologías al servicio de los niños, niñas, jóvenes y adultos en beneficio de la recuperación psicoafectiva y la salud mental. 


\section{Conclusiones}

Una sociedad hipermoderna plantea nuevos desafíos a las ciencias sociales y humanas. La vida virtual, las tecnologías y las exigencias del día a día generan nuevas formas de relación mediadas por los dispositivos tecnológicos, y escasez en el tiempo para lidiar con el estrés cotidiano, el trabajo y la vida social. "Los acontecimientos de la era postmoderna se reflejan como organizaciones de lo efímero, en virtud de pensamientos, creencias, ideologías y formas en las que el hombre concibe su vida social" (Mass, 2013, p. 160), y evidencian nuevas complejidades en los individuos.

Las problemáticas sociales, políticas o económicas producen tensiones en las personas que conducen a alteraciones de la salud mental tanto en adultos como en niños/as y adolescentes. Es decir, que todo ser humano se encuentra involucrado con un entorno social que acrecienta sus demandas de producción (por ejemplo, el trabajo), lo que no es ajeno a las condiciones que facilitan trastornos mentales y diversas patologías que afectan la salud de individuos y poblaciones.

Es la salud mental un reto mundial y nacional, en lo referido a la promoción y prevención, pero es en materia de detección temprana e intervención que se requieren nuevos esfuerzos por atender las consecuencias de las tensiones generadas por los desafíos a los que se enfrentan los individuos diariamente, en particular, la infancia, y las interferencias en el desarrollo.

Condiciones sociales desfavorables, durante la infancia, entorpecen el desarrollo esperado, y ponen a prueba los recursos afectivos y relacionales de los niños/as, su inmadurez constitucional requiere mayor atención, pues aún se encuentran en perfeccionamiento las dimensiones del desarrollo psicoafectivo. Y como advierten Tamez et. al (2006) cuando las interferencias "no son resueltas favorablemente generan estados de regresión y de fijación que pueden dar como resultado estructuras psicopatológicas" (p. 16). Por tanto, la recuperación psicoafectiva es una urgencia en poblaciones vulnerables.

En Colombia la vulnerabilidad está definida por el Departamento de Planeación Nacional (2007) citando a Moser "como la inseguridad y sensibilidad del bien-estar (well-being) de los individuos, hogares y 
comunidades en situaciones de cambio, e implícitamente en su respuesta y resiliencia a los riesgos que ellos enfrentan durante estos cambios negativos" (p. 5). Por su parte, el Banco Mundial (2000) sugiere que la vulnerabilidad se mide de acuerdo con "la capacidad de resistencia ante una crisis [y] la probabilidad de que una crisis dé por resultado una disminución del bienestar" (p. 139). En el caso de la infancia, el impacto de las interferencias del desarrollo, supera la capacidad de sobreponerse a sus efectos, pues su condición vital, o sea, su inmadurez, es en esencia vulnerable.

La vulnerabilidad parece ser un factor asociado a las interferencias en el desarrollo, y como consecuencia, una mala salud. Pues, "un ambiente suficientemente bueno es, podría decirse, el que favorece las diversas tendencias individuales heredadas de modo tal que el desarrollo se produce conforme a esas tendencias" (Winnicott, 1967, p. 28). En este sentido, la recuperación psicoafectiva, a través de la creación de dispositivos de elaboración psíquica, favorece el desarrollo psicoafectivo y la salud mental, al asistir el carácter integral del desarrollo.

Se hace necesaria la implementación de programas de recuperación psicoafectiva, que permitan restaurar la capacidad de integración de los niños y niñas, desde el impulso creador, las funciones del yo libre de conflicto y su relación con el ambiente. Es vital “(...) la educación emocional y el desarrollo psicoafectivo como una respuesta que permite prevenir problemáticas psicosociales, y promocionar la salud psicoemocional del individuo, de manera lúdico-educativa" (Programa Pisotón, 2015).

Si un programa es "un todo coherente, organizado y estructurado con objetivos, medios y personas que los dirigen. Se justifica tomando como base las necesidades definidas como una carencia o una falta que afecta a los individuos, una comunidad o una sociedad" (Plante, citado por Ridde y Dagenais, 2015, p. 21), su propósito se constituye en "lograr una salud mental positiva, mejorar la calidad de vida" (Hosman y Jané-Llopis, 1999, p. 31), en este caso, en niños/as en interferencias en el desarrollo.

Así, un programa para la recuperación psicoafectiva, de orientación dinámica, se corresponde al "ambiente facilitador [que] es necesario, y si no es suficientemente bueno, el proceso madurativo se debilita o decae" (Winnicott, 1963, p. 86). Es decir que, actúa desde el proceso madurativo-constitucional y relacional, pues, cuanto más positivas resultan las condiciones ambientales, más óptimamente ocurre el desarrollo 
psicoafectivo. Así pues, un programa de intervención psicológica para la recuperación psicoafectiva de orientación dinámica debería contar con las siguientes condiciones:

a. Comprender de las condiciones madurativo-constitucionales y relacionales derivadas del ciclo vital humano.

b. Conocer las consecuencias biopsicosociales de las interferencias en el desarrollo en los afectados.

c. Estar basados en técnicas lúdico-educativas y su relación con el ciclo vital.

d. Fomentar el despliegue del desarrollo psicoafectivo a través de acciones, estrategias y actividades que permitan el reconocimiento y elaboración de las interferencias en el desarrollo.

e. Propiciar la expresión emocional de los niños/as, y su resignificación.

f. Incluir a los padres y maestros en las actividades del programa, de modo que se fortalezcan vínculos afectivos saludables.

La prioridad de la recuperación psicoafectiva se dirige al desarrollo integral pues "lo que constituye la base de la salud mental es la culminación de esos procesos" (Winnicott, 1969, p. 93), de los procesos biopsicosociales y emocionales en la infancia que ocupan lo fundamental de la personalidad y la integración emocional, o sea la salud mental.

Si se reconoce que "la exposición a las adversidades a edades tempranas es un factor de riesgo prevenible bien establecido de los trastornos mentales" (OMS, 2013, p. 7), entonces, la recuperación psicoafectiva es un compromiso ético y disciplinar que se sustenta en "promover la recuperación física y psicológica y la reintegración social de todo niño víctima..." (UNICEF, 1989, artículo 39, p. 36). Y como advierten Amar, Madariaga y Macías (2014) en el caso de recuperación psicoafectiva, se requiere "garantizar tratamiento psicoafectivo a los niños que hayan sido víctimas de un conflicto armado, tortura, abandono, maltrato y explotación para que puedan lograr su recuperación y reintegración social" (p. 34). O sea, en restablecer las dimensiones del desarrollo integral, o sea, lo biopsicosocial. 


\section{Referencias}

Amar, J., Madariaga, C. y Macías A. (2014). Infancia, familia y derechos humanos. Barranquilla: Editorial Universidad del Norte.

Ávila, A. (2009). La psicoterapia psicoanalítica relacional: Conceptos fundamentales y perspectivas. Recuperado de: http://www. psicoterapiarelacional.es/Portals/0/Documentacion/AAvila/A_ Avila_Psicoterapia-psicoanalitica-relacional_Interpsiquis_2009.pdf

Banco Mundial. (2000). Informe sobre el desarrollo mundial. 2000/2001. Lucha contra la pobreza. Recuperado de http://documentos.bancomundial.org/curated/es/509031468137396214/pdf/226840SPANISHOWDR0200002001.pdf.

Bernal, C. (2010). Metodología de la investigación. Editorial Pearson.

Bowlby, John y World Health Organization. (1951). Maternal care and mental health: a report prepared on behalf of the World Health Organization as a contribution to the United Nations programme for the welfare of homeless children / John Bowlby, 2nd ed. Geneva : World Health Organization. http://www.who.int/iris/handle/10665/40724.

Blos, P. (2011). Los comienzos de la adolescencia. Buenos Aires: Amorrortu Editores.

Bustos, V. \& Russo, A. (2018). Salud mental como efecto del desarrollo psicoafectivo en la infancia. Psicogente, 21(39), 183-202. http:// doi.org/10.17081/psico.21.39.2830.

Cárcamo, C. (1993). Prefacio. En: El yo y los mecanismos de defensa. 9-10. Buenos Aires: Editorial Paidós.

Cohen, S. (2015). La niñez cautiva. Salud mental infantil y juvenil. México: Fondo de Cultura Económica.

De La Fuente, R. (2014). Introducción a la salud mental. En: Salud mental y medicina psicológica. 2-5. México: McGraw-Hill.

DPN (2007). Departamento de Planeación Nacional. Una aproximación a la vulnerabilidad. Recuperado de: https://colaboracion.dnp.gov.co/ cdt/desarrollo\%20social/boletin34_1.pdf. 
Duro, E. (2012). Prologo. En: Desarrollo emocional. Clave para la primera infancia. UNICEF\&KALEIDOS. Recuperado de: https://www.Unicef.org/ecuador/Desarrollo_emocional_0a3_simples.pdf.

Fossa, P. (2013). La desorganización psíquica en el desarrollo infantil. Revista GEPU 4 (1), 150- 159. Recuperado de: http://revistadepsicologiagepu.es.tl/Vol-.--4-No-.--1.htm.

Freud, A. (1977). Psicoanálisis del niño. Argentina: Ediciones Hormé.

Freud, A. (1979) Normalidad y patología en la niñez. Argentina: Editorial Paidós psicología profunda.

Freud, A. (1993). El yo y los mecanismos de defensa. Buenos Aires: Editorial Paidós.

Gómez, E. (2013). Trauma relacional temprano. Chile: Ediciones Universidad Alberto Hurtado.

Greenspan, S. (1992). Infancy and Early childhood: The practice of clinical assessment and intervention with emotional and developmental challenges. Madison: International University Press.

Guillerault, G. (2009). Dolto/Winnicott. El bebé en psicoanálisis. Buenos Aires: Editorial Paidós.

Hosman C. y Jané-Llopis E. (1999). Retos politicos2: Salud mental. En: La evidencia de la efectividad de la promoción para la salud: conformando la salud publica en una nueva Europa. Bruselas, ECSC-ECEAEC:29-41.

Klein, M. (1960). Sobre la salud mental. En: Envidia y gratitud. Y otros trabajos. 272-278. Argentina: Editorial Paidós.

Laplanche, J; Pontalis, J. (1996). Diccionario de Psicoanálisis. Buenos Aires. Editorial Paidós.

Mass, L.R. (2013). El sujeto y la estética corporal en la sociedad contemporánea (algunas relaciones teóricas con el capitalismo y plus de gozar). Revista Psicogente, vol. 17 No.31; 155-162. Recuperado de: http://publicaciones.unisimonbolivar.edu.co:82/rdigital/psicogente/index.php/psicogente/article/viewFile/428/393m.

Minsalud. (2015). Encuesta Nacional de Salud Mental. Tomo I. Ministerio de Salud y Protección Social. Recuperado de https://www.javerianacali.edu.co/sites/ujc/files/node/field-documents/field_document_ file/saludmental_final_tomoi_color.pdf. 
Oblitas, L. (2004). Psicología de la salud y calidad de vida. México: Thompson.

OMS .(2001). Fortaleciendo la promoción de la salud mental. Ginebra. Organización mundial de la salud. Hoja informativa. Nro. 220.

OMS .(2004). Prevención de los Trastornos Mentales. Intervenciones efectivas y opciones de políticas. Informe compendiado. Un Informe del Departamento de Salud Mental y Abuso de Sustancias de la Organización Mundial de la Salud, en colaboración con el Centro de Investigación de Prevención de las Universidades de Nijmegen y Maastricht. Organización Mundial de la Salud. Ginebra. Recuperado de: http://apps.who.int/iris/bitstream/10665/78545/1/924159215X_ spa.pdf.

OMS. (2013). Plan de acción para la salud mental 20132020. Recuperado de: http://apps.who.int/iris/bitstre am/10665/97488/1/9789243506029_spa.pdf.

OMS. (2018). Salud mental del adolescente.

. Recuperado de: https://www.who.int/es/news-room/fact-sheets/detail/ adolescent-mental-health.

Packer, M. (2013). La ciencia de la investigación cualitativa. Ediciones Uniandes.

Piñuel, J. (2002). Epistemología, metodología y técnicas del análisis del contenido. Revista Estudios de Sociolingüística, 3(1), 1-42. Recuperado: https://www.researchgate.net/publication/267797356_Epistemologia_metodologia_y_tecnicas_d el_analisis_de_contenido.

Phillips, A. (1998). Winnicott. Buenos Aires: Lugar Editorial.

Programa Pisotón. (2015). Programa de Educación y Desarrollo Psicoafectivo. Recuperado de: http://www.uninorte.edu.co/web//pisoton-uninorte.

Rangel, M. (2009). Psicoterapia infantil. Un enfoque psicoanalítico. México: Editorial Trillas.

Ridde, V. y Dagenais, C. (2015). Introducción general a la evaluación de programas. En: Enfoques y prácticas en la evaluación de programas.17-34. Bogotá: Editorial Pontificia Universidad Javeriana.

Romo, F. y Patiño, L. (2014). Ciclo vital y salud mental. En: Salud mental y medicina psicológica. 2-5. México: McGraw-Hill. 
Serguéyevna, V. (2015). Psicología evolutiva. España: Machado Nuevo Aprendizaje.

Strauss, A; Corbin, J. (2012). Bases de la investigación cualitativa. Técnicas y procedimientos para desarrollar la teoría fundamentada. Editorial Universidad de Antioquia.

Tamez, R., Ortega S., Roa, A., Russo A. y Galindo, J. (2006). Educación y desarrollo psicoafectivo. Barranquilla, Ediciones Uninorte.

UNICEF (1989). Convención sobre los derechos del niño. Recuperado de: http://www.Unicef.org/argentina/spanish/7.-Convencionsobrelosderechos.pdf.

UNICEF. (2004). Estado mundial de la infancia 2005. La infancia amenazada. Recuperado de: https://www.Unicef.org/spanish/sowc05/ sowc05_sp.pdf.

UNICEF. (2012). Estado Mundial De La Infancia. Informe Ejecutivo. Recuperado de: http://www.Unicef.org/spanish/sowc2012/pdfs/ SOWC\%202012\%20Executive\%20Summary\%20LoRes\%20PDF_ SP_03132012.pdf.

UNICEF (2014). El estado mundial de la infancia de 2014 en cifras. Todos los niños cuentan. Recuperado de: http://www.Unicef.org/spanish/ sowc2014/numbers/.

Uribe, J. (2013). La investigación documental y el estado del arte como estrategias de investigación en ciencias sociales. En La investigación en Ciencias Sociales: Estrategias de investigación (pp. 197-212). Bogotá: Universidad Piloto de Colombia.

Valbuena, E. (2013). El análisis del contenido: De lo manifiesto a lo oculto. En La investigación en Ciencias Sociales: Estrategias de investigación (pp. 213-224). Bogotá: Universidad Piloto de Colombia.

Winnicott, D. (1951). Las bases de la salud mental. En: Deprivación y delincuencia. 197-200. Buenos Aires: Editorial Paidós.

Winnicott, D. (1961). Psicoanálisis y ciencia: ¿amigos o parientes? En: El hogar, nuestro punto de partida. Ensayos de un psicoanalista. 17-23. Buenos Aires: Editorial Paidós.

Winnicott, D. (1963). El valor de la depresión. En: El hogar, nuestro punto de partida. Ensayos de un psicoanalista. 84-93. Buenos Aires: Editorial Paidós. 
Winnicott, D. (1965). El niño en el grupo familiar. En: El hogar, nuestro punto de partida. Ensayos de un psicoanalista. 149-163. Buenos Aires: Editorial Paidós.

Winnicott, D. (1967). El concepto de individuo sano. En: El hogar, nuestro punto de partida. Ensayos de un psicoanalista. 27-47. Buenos Aires: Editorial Paidós.

Winnicott, D. (1968). Conceptos contemporáneos sobre el desarrollo adolescente, y las inferencias que de ellos se desprende en lo que respecta a la educación superior. En: Realidad y juego. 215-232. España: Editorial Gedisa.

Winnicott, D. (1969). De la pédiatrie á la psychanalyse. Paris: Payot.

Winnicott, D. (1970a). Vivir creativamente. En: El hogar, nuestro punto de partida. Ensayos de un psicoanalista. 48-65. Buenos Aires: Editorial Paidós.

Winnicott, D. (1970b). Processus de maturation chezl'enfant. Paris: Payot. 